TRANSACTIONS OF THE

AMERICAN MATHEMATICAL SOCIETY

Volume 350, Number 3, March 1998, Pages 889-912

S 0002-9947(98)01666-3

\title{
ALGEBRAIC TRANSITION MATRICES IN THE CONLEY INDEX THEORY
}

\author{
ROBERT FRANZOSA AND KONSTANTIN MISCHAIKOW
}

\begin{abstract}
We introduce the concept of an algebraic transition matrix. These are degree zero isomorphisms which are upper triangular with respect to a partial order. It is shown that all connection matrices of a Morse decomposition for which the partial order is a series-parallel admissible order are related via a conjugation with one of these transition matrices. This result is then restated in the form of an existence theorem for global bifurcations. Simple examples of how these results can be applied are also presented.
\end{abstract}

\section{INTRODUCTION}

In classical Morse theory the gradient vector field of a Morse function on a compact manifold gives rise to a flow with a finite number of hyperbolic fixed points. C. Conley introduced a generalization of this in the form of a Morse decomposition. To be more precise, let $\varphi: \mathbf{R} \times X \rightarrow X$ be a continuous flow on a locally compact Hausdorff space ${ }^{1}$ and let $S$ be a compact invariant set under $\varphi$. A Morse decomposition of $S$ is a collection of mutually disjoint compact invariant subsets of $S$,

$$
\mathcal{M}(S):=\{M(p) \mid p \in \mathcal{P}\}
$$

indexed by a finite set $\mathcal{P}$, on which it is possible to impose a partial order $<$ such that if $x \in S \backslash \bigcup_{p \in \mathcal{P}} M(p)$, then there exists $q<p$ such that $\alpha(x) \subset M(p)$ and $\omega(x) \subset M(q)$. The individual sets $M(p)$ are called Morse sets. Any partial order on $\mathcal{P}$ which satisfies the above mentioned properties is called an admissible order. Because of its inherent flexibility (any compact invariant set may be a Morse set) this decomposition has been used in a variety of settings $[9,4,11,18,20,31]$.

Of course, the power of Morse theory comes from its ability to relate local information (the Morse indices of the hyperbolic critical points) to global information (the homology of the compact manifold). This relation appears in the form of the Morse inequalities. In the Conley theory one begins with the Conley index for

Received by the editors January 3, 1995 and, in revised form, October 4, 1995.

1991 Mathematics Subject Classification. Primary 58F35; Secondary 58F30, 35K57.

Key words and phrases. Conley index, connection matrix, transition matrix, bistable attractor, travelling waves.

Research was supported in part by NSF Grant DMS-9101412. Part of this paper was written while the second author was visiting the Instituto de Ciencias Mathematicas de São Carlo of the Universidade de São Paulo. He would like to take this opportunity to thank the members of the institute for their warm hospitality.

${ }^{1}$ All the theoretical results of this paper can be carried out in the setting of admissible semiflows on metric spaces. For the background on the index theory in this setting the reader is referred to the work of K. Rybakowski [29]. 
isolated invariant sets. Recall that given the flow $\varphi, S \subset X$ is an isolated invariant set if there exists a compact set $N \subset X$ such that

$$
S=\operatorname{Inv}(N, \varphi):=\{x \in N \mid \varphi(\mathbf{R}, x) \subset N\} \subset \operatorname{int} N .
$$

The Conley index of $S$, denoted by $h(S)$, is the homotopy type of a pointed space, i.e.

$$
h(S) \sim\left(Y, y_{0}\right) .
$$

For computational purposes it is more convenient to use the homological version of the index

$$
C H_{*}(S):=H_{*}\left(Y, y_{0}\right) .
$$

In the Conley theory, the Morse inequalities are replaced by Conley's connection matrix. The basic results for this theory can be found in $[5,6,7,8]$. For the moment we remark that given a Morse decomposition, a corresponding connection matrix is a linear map

$$
\Delta: \bigoplus_{p \in \mathcal{P}} C H_{*}(M(p)) \rightarrow \bigoplus_{p \in \mathcal{P}} C H_{*}(M(p))
$$

which is both a boundary operator $(\Delta \circ \Delta=0$ and $\Delta$ is a degree -1 operator with respect to the grading on the indices of the Morse sets) and strictly upper triangular with respect to any admissible ordering (if $p \nless q$, then the entries in $\Delta$ mapping $\mathrm{CH}_{*}(\mathrm{M}(q))$ to $\mathrm{CH}_{*}(M(p))$ are necessarily zero). An important feature, which plays a prominent role in this paper, is that connection matrices need not be unique.

The Conley theory has an essential feature which distinguishes it from Morse theory and that is the continuation property of the index. Let us recall its definition. Let $\varphi_{\lambda}: \mathbf{R} \times X \rightarrow X, \lambda \in \Lambda$, denote a continuous family of flows parametrized by a connected, locally contractible, locally path connected space $\Lambda$. This is equivalent to the continuity of the parametrized flow

$$
\Phi: \mathbf{R} \times X \times \Lambda \rightarrow X \times \Lambda
$$

defined by

$$
\Phi(t, x, \lambda)=\left(\varphi_{\lambda}(t, x), \lambda\right) .
$$

Of course, given any $U \subset \Lambda, \Phi$ restricts to a parametrized flow

$$
\Phi_{U}: \mathbf{R} \times X \times U \rightarrow X \times U .
$$

Let $N \subset X \times \Lambda$. By definition $N$ is an isolation neighborhood for $\Phi$ if

$$
\operatorname{Inv}(N, \Phi) \subset \operatorname{int} N
$$

Let $N_{\lambda}:=N \cap(X \times\{\lambda\})$. Observe that if $N$ is an isolation neighborhood for $\Phi$, then for all $\lambda \in \Lambda, N_{\lambda}$ is an isolation neighborhood for $\varphi_{\lambda}$. Two isolated invariant sets $S_{\lambda_{0}}$ and $S_{\lambda_{1}}$ for the flows $\varphi_{\lambda_{0}}$ and $\varphi_{\lambda_{1}}$, respectively, are said to be related by continuation if there exist a path connected set $U \subset \Lambda$ such that $\lambda_{0}, \lambda_{1} \in U$ and an isolating neighborhood $N$ of $\Phi_{U}$ such that $S_{\lambda_{0}}=\operatorname{Inv}\left(N_{\lambda_{0}}, \varphi_{\lambda_{0}}\right)$ and $S_{\lambda_{1}}=\operatorname{Inv}\left(N_{\lambda_{1}}, \varphi_{\lambda_{1}}\right)$. A fundamental fact is that in this case

$$
h\left(S_{\lambda_{0}}\right) \sim h\left(S_{\lambda_{1}}\right) .
$$

In other words, the Conley index is preserved under continuation. 
The definition of continuation of Morse decompositions is obtained in a similar manner. To be precise, let $U \subset \Lambda$ be connected, let $S$ be a compact invariant set, and let $\{M(p) \mid p \in \mathcal{P}\}$ be a finite collection of disjoint compact invariant subsets with respect to the parametrized flow $\Phi_{U}$. These, of course, give rise to parametrized families of invariant sets $S_{\lambda}$ and $M_{\lambda}(p)$, for $\lambda \in U$, obtained by intersecting with the fibers $X \times\{\lambda\}$. The Morse decomposition is said to continue over $U$, if for each $\lambda \in U$,

$$
\mathcal{M}\left(S_{\lambda}\right)=\left\{M_{\lambda}(p) \mid p \in \mathcal{P}\right\}
$$

is a Morse decomposition of $S_{\lambda}$.

An important point which needs to be made is that this definition is weaker than assuming that

$$
\mathcal{M}(S)=\{M(p) \mid p \in \mathcal{P}\}
$$

is a Morse decomposition for $S$ under $\Phi_{U}$. In this latter case, there exists an admissible partial order $<$ on $\mathcal{P}$ which is an admissible order for each Morse decomposition $\mathcal{M}\left(S_{\lambda}\right)=\left\{M_{\lambda}(p) \mid p \in \mathcal{P}\right\}$. However, assuming that we have a Morse decomposition for each parameter value does not insure the existence of an admissible partial order for $\mathcal{M}(S)$. In other words, the existence of admissible orders $<_{\lambda}$ for each $\lambda \in \Lambda$ does not guarantee that these orders are compatible. As shall be seen in Section 5.1, the results of this paper can be used, in some instances, to determine when a local admissible order is, in fact, global. We shall refer to this stronger notion of continuation as follows. Two Morse decompositions $\mathcal{M}\left(S_{\lambda_{0}}\right)$ and $\mathcal{M}\left(S_{\lambda_{1}}\right)$ are related by an order preserving continuation if there exists a path connected set $U \subset \Lambda$ containing $\lambda_{0}$ and $\lambda_{1}$ such that $\mathcal{M}(S)$ is a Morse decomposition for $\Phi_{U}$. Equivalently, we shall say that $\mathcal{M}(S)$ continues with order over $U$. From the local continuation theorem for Morse decompositions [7] we obtain the following theorem.

Theorem 1.1. Let $K \subset \Lambda$ be compact. Assume that $\mathcal{M}(S)$ continues over $K$. Then, there exists a finite covering $\left\{U_{i} \mid i=1, \ldots, I\right\}$ of $K$ such that $\mathcal{M}(S)$ continues with order over each $U_{i}$.

Changing our train of though for a moment, consider a Morse decomposition $\mathcal{M}(S)=\{M(p) \mid p \in \mathcal{P}\}$ and assume that $<$ is not an admissible order. The simplest way that this can occur is for there to exist an $x \in S$ and $p, q \in \mathcal{P}$ with $q<p$, $\omega(x) \subset M(p)$, and $\alpha(x) \subset M(q)$. Thus the set of admissible orderings provides information concerning the existence of connecting orbits between Morse sets.

Returning to the setting of a Morse decomposition $\mathcal{M}\left(S_{\lambda}\right)=\left\{M_{\lambda}(p) \mid p \in \mathcal{P}\right\}$ which continues over a parameter space $\Lambda$, let $\mathcal{C} \mathcal{M}\left(\mathcal{M}\left(S_{\lambda_{0}}\right)\right)$ and $\mathcal{C} \mathcal{M}\left(\mathcal{M}\left(S_{\lambda_{1}}\right)\right)$ denote the set of connection matrices for $\mathcal{M}\left(S_{\lambda_{0}}\right)$ and $\mathcal{M}\left(S_{\lambda_{1}}\right)$, respectively. Since the individual Morse sets continue over $\Lambda$,

$$
C H_{*}\left(M_{\lambda_{0}}(p)\right) \approx C H_{*}\left(M_{\lambda_{1}}(p)\right),
$$

and hence, the connection matrices are defined on isomorphic linear spaces. Therefore, if $\mathcal{C} \mathcal{M}\left(\mathcal{M}\left(S_{\lambda_{0}}\right)\right)$ and $\mathcal{C M}\left(\mathcal{M}\left(S_{\lambda_{1}}\right)\right)$ differ, one may expect that the set of admissible partial orders at $\lambda_{0}$ and $\lambda_{1}$ are different. Furthermore, parameter values at which the sets of admissible partial orders change must correspond to the existence of global bifurcations.

The ultimate goal of our project is to develop an algebraic means of proving the existence of and understanding the dynamic implications of these bifurcation 
points. Unfortunately we are still a long way from attaining this, and in this paper we can only present some preliminary (but nontrivial) results in this direction. Since the results are fairly abstract we shall attempt to put them into perspective by discussing two alternative methods which have proven useful in a variety of contexts.

The idea of the first approach was due to Conley and carried out in a special setting by J. Reineck [26] (see also [16, 17]). C. McCord and the second author [15] recently abstracted these ideas and dubbed this approach the method of singular transition matrices. It is most easily described in the context developed by Reineck, i.e. that of a parametrized family of ordinary differential equations defined on $\mathbf{R}^{n}$,

$$
\dot{x}=f(x, \lambda),
$$

where the parameter space $\Lambda$ is the set of real numbers $\mathbf{R}$. For the sake of simplicity, assume that the Morse decomposition $\mathcal{M}\left(S_{\lambda}\right)=\left\{M_{\lambda}(p) \mid p \in \mathcal{P}\right\}$ continues over $\mathbf{R}$ and that connection matrices $\Delta_{-1}$ and $\Delta_{1}$ for the Morse decompositions $\mathcal{M}\left(S_{-1}\right)$ and $\mathcal{M}\left(S_{1}\right)$, respectively, are known. Furthermore, let $N \subset \mathbf{R}^{n}$ be an isolation neighborhood for $S_{\lambda}, \lambda \in \mathbf{R}$.

In order to understand the bifurcations for intermediate parameter values, i.e. for $-1<\lambda<1$, Conley suggested introducing slow dynamics in the parameter space as follows:

$$
\dot{x}=f(x, \lambda), \quad \dot{\lambda}=\varepsilon\left(\lambda^{2}-1\right)
$$

where, for the moment, we assume $\varepsilon>0$. Define

$$
M\left(p^{+}\right):=M_{1}(p), \quad M\left(p^{-}\right):=M_{-1}(p)
$$

where $M\left(p^{ \pm}\right)$is taken to be the obvious subset in $\mathbf{R}^{n} \times\{ \pm 1\} \subset \mathbf{R}^{n} \times \Lambda$. For $\varepsilon>0$ sufficiently small, $N \times[-2,2]$ is an isolating neighborhood for the flow $\Phi_{\varepsilon}$ generated by (2) [16]. Let $K_{\varepsilon}:=\operatorname{Inv}\left(N \times[-2,2], \Phi_{\varepsilon}\right)$. Now observe that since $\dot{\lambda}<0$ if $\lambda \in(-1,1)$, for all $\varepsilon>0$,

$$
\mathcal{M}\left(K_{\varepsilon}\right)=\left\{M\left(p^{ \pm}\right) \mid p \in \mathcal{P}\right\}
$$

is a Morse decomposition, and furthermore, there is an admissible ordering given by

$$
\begin{aligned}
& q^{-}<p^{+}, \\
& q^{-}<p^{-} \Leftrightarrow q<_{-1} p, \\
& q^{+}<p^{+} \Leftrightarrow q<_{1} p,
\end{aligned}
$$

where $<_{-1}$ and $<_{1}$ are admissible orders for $\mathcal{M}\left(S_{-1}\right)$ and $\mathcal{M}\left(S_{1}\right)$, respectively. Let $\Delta_{\varepsilon}$ denote a connection matrix for $\mathcal{M}\left(K_{\varepsilon}\right)$. Then, since the dynamics on the subspaces $\mathbf{R}^{n} \times\{ \pm 1\}$ is given exactly by the flows generated by $\dot{x}=f(x, \pm 1)$, it is not surprising that

$$
\begin{aligned}
\Delta_{\varepsilon}: & \bigoplus_{p \in \mathcal{P}} C H_{*}\left(M\left(p^{-}\right)\right) \bigoplus_{p \in \mathcal{P}} C H_{*}\left(M\left(p^{+}\right)\right) \\
& \rightarrow \bigoplus_{p \in \mathcal{P}} C H_{*}\left(M\left(p^{-}\right)\right) \bigoplus_{p \in \mathcal{P}} C H_{*}\left(M\left(p^{+}\right)\right)
\end{aligned}
$$

takes the form

$$
\Delta_{\varepsilon}=\left[\begin{array}{cc}
\Delta_{-} & T_{\varepsilon} \\
0 & \Delta_{+}
\end{array}\right]
$$


where $\Delta_{ \pm}$are connection matrices for $\mathcal{M}\left(S_{ \pm 1}\right)$. Observe that we said "takes the form" rather than "equals". This is necessary since the space on which $\Delta_{\varepsilon}$ is defined is not the direct sum of the spaces on which $\Delta_{ \pm}$are defined. One of Reineck's contributions [26] was to formalize the expression (3). It is possible to make sense of the limit of $T_{\varepsilon}$ as $\varepsilon \rightarrow 0$, and the resulting matrices are referred to as the singular transition matrices [15]. In [26] it is shown that a nonzero element of a singular transition matrix implies the existence of connecting orbits between appropriate Morse sets for some parameter value $\lambda \in(-1,1)$. Thus, these transition matrices can be used to detect global bifurcations.

This form of transition matrix is by far the most general. In fact, it is sufficient to assume the existence of an isolated invariant set that continues over $\Lambda$ and the existence of Morse decompositions of this invariant set at two different parameter values. However, since these transition matrices are essentially just submatrices of a connection matrix, they can only be computed via the dynamics of the slow system (2).

Actually, this last statement is a little too strong. First, as is shown in [24], knowledge of the dynamics of the parametrized family (1) can be used to compute $T$; however, if the goal is to understand the dynamics of the parametrized family this information is of little use.

Second, if we assume that the Morse decomposition continues over the parameter space, then there are algebraic constraints on $T$. Since $\Delta_{\varepsilon}$ is a connection matrix, $T$ must be both upper triangular with respect to the admissible order $<$ and a degree -1 operator. Furthermore, it can be shown that $T$ is an isomorphism. Finally, since $\Delta_{\varepsilon} \circ \Delta_{\varepsilon}=0$, the following matrix equation must be satisfied:

$$
\Delta_{-} T+T \Delta_{+}=0 .
$$

Of course, (4) is merely a sufficient condition, and hence, can only be used to rule out possible transition matrices. In the extreme case in which $\Delta_{ \pm}=-0$, (4) provides no information. Finally, even though $T$ may be a well defined linear map, its algebraic properties are not explained through this construction.

In an attempt to better understand singular transition matrices and for the purpose of applications in which (4) provided little information, C. McCord and the second author developed a topological transition matrix [13]. The starting point for this theory is a refinement of the homotopy Conley index, known as a connected simple system. This consists of the Conley index $h(S)$ and the homotopy type of all flow induced maps on $h(S)$. Under the assumption of an order preserving continuation of the Morse decomposition over $\Lambda$ and using the homology functor to pass from $h(\cdot)$ to $C H_{*}(\cdot)$, the continuation theorem was used to define a basis for $C H_{*}\left(M_{\lambda}(p)\right)$ for each $\lambda \in \Lambda$ and $p \in \mathcal{P}$. Now under the assumption that at the parameter values $\lambda_{0}$ and $\lambda_{1}$ for which the dynamics is understood $S_{\lambda_{0}}=\bigcup M_{\lambda_{0}}(p)$ and $S_{\lambda_{1}}=\bigcup M_{\lambda_{1}}(p)$, i.e. that there are no connecting orbits, the difference between the local isomorphisms (i.e between the Morse sets at $\lambda_{0}$ and $\lambda_{1}$ ) and the global isomorphism (the isomorphism between the index of $S$ at $\lambda_{0}$ and $\lambda_{1}$ ) defines a matrix called the topological transition matrix. Formally, this matrix satisfies similar properties to those of the singular transition matrices. In particular, it is potentially nontrivial between the same vector spaces as the singular matrix and it is an isomorphism. Since it is assumed that there are no connections at the parameter values $\lambda_{0}$ and $\lambda_{1}, \Delta_{\lambda_{0}}$ and $\Delta_{\lambda_{1}}$ are identically 0 , and hence formally (4) is satisfied. 
Finally, off diagonal nonzero entries of this transition matrix, again, imply the existence of connecting orbits between Morse sets for flows at intermediate parameter values.

In this case, however, the construction of the matrix is done via algebraic topology. Hence, the algebraic properties of topological transition matrices are clear. In particular, if we let $T_{\lambda_{1}, \lambda_{0}}$ denote the topological transition matrix from $\lambda_{0}$ to $\lambda_{1}$, and assume that the parameter space is simply connected, then, for example,

$$
T_{\lambda_{2}, \lambda_{0}}=T_{\lambda_{2}, \lambda_{1}} \circ T_{\lambda_{1}, \lambda_{0}}
$$

and

$$
T_{\lambda_{1}, \lambda_{0}}=T_{\lambda_{0}, \lambda_{1}}^{-1} .
$$

It is reasonable at this point to ask whether these latter properties are of any interest. The answer is yes and worth explaining since it justifies the need for the algebraic machinery we are attempting to develop. (5) was used in [13] to establish the existence of global bifurcations. This result, in turn, was exploited to prove the existence of specific travelling waves for a general family of predatorprey systems [25]. In both of these cases the connection matrices at the known parameter values were identically 0 , and hence (4) was of no use in determining the singular transition matrices. (6) has been used for a singular perturbation problem. In [10] information concerning a parametrized family of equations of the form (1) was assumed and knowledge of connecting orbits for a system of the form (2) was obtained. In particular, for $\varepsilon>0$ the dynamics is sufficiently simple to compute the singular transition matrix. However, for $\varepsilon<0$, the case of interest, the dynamics is too complicated to study directly. In [14] it is shown that when both topological and singular matrices can be defined, then they both agree. Therefore, for $\varepsilon<0$ the singular transition matrix is the inverse of the singular transition matrix for $\varepsilon>0$.

The reason for including these two examples was to emphasize the importance of having an algebraic understanding of the transition matrix. Unfortunately, the results of [14] only hold in rather restrictive settings. The purpose of this paper is to obtain yet another transition matrix which we refer to as an algebraic transition matrix which is defined in a reasonably general context, i.e. whenever one has an order preserving continuation of a Morse decomposition. Of course definitions are easy to make; however, we also show that these matrices can be used to generate all connection matrices at a particular parameter value (thus addressing in part the nonuniqueness question) and that these matrices can be used to detect global bifurcations. We leave it to future papers to explore the relationship between algebraic, topological, and singular transition matrices.

This paper is organized as follows. In Section 2, the necessary background on partial orders is presented. Section 3 contains the essential algebraic results. In Section 4 the algebraic results of Section 3 are recast in the form of theorems related to global bifurcations and the algebraic transition matrices are defined. Finally, in the last section, we discuss some simple applications of our results to differential equations. 


\section{Partial orders}

We assume that the reader is familiar with topics from the theory of partial orders as in [5]. Throughout the paper $P$ represents a finite set with a partial order $<$. If $Q$ is a subset of $P$, the $<_{Q}$ denotes the partial order $<$ restricted to $Q$.

An interval in $P$ is the set $I \subset P$ which is such that if $p, q \in I$ and $p<r<q$ then $r \in I$. The set of intervals in $<$ is denoted by $I(<)$. An adjacent $n$-tuple of intervals in $<$ is an $n$-tuple of mutually disjoint nonempty intervals, $\left(I_{1}, \ldots, I_{n}\right)$, whose union is an interval in $<$ and which are such that if $p \in I_{i}, q \in I_{j}$, and $p<q$, then $i<j$. The set of adjacent $n$-tuples of intervals in $<$ is denoted by $I_{n}(<)$. An adjacent 2-tuple of intervals is also called an adjacent pair of intervals. If $\left(I_{1}, \ldots, I_{n}\right)$ is an adjacent $n$-tuple of intervals whose union is $I$, then $\left(I_{1}, \ldots, I_{n}\right)$ is called a decomposition of $I$, if $A, B \subset P$ are disjoint then we say that $A$ and $B$ are noncomparable if neither $a<b$ nor $b<a$ for every $a \in A, b \in B$, and we say that $B$ is totally greater than $A$ if $a<b$ for every $a \in A$ and $b \in B$.

A partial order $<$ on $P$ is called stackable if there is a decomposition of $P$, $\left(I_{1}, \ldots, I_{n}\right)$, such that $<$ restricted to each $I_{i}$ is trivial and such that if $i<j$ then $I_{j}$ is totally greater than $I_{i}$. Note that a trivial order is stackable and a total order is stackable.

In Section 3 an inductive proof of the existence of a matrix that is upper triangular with respect to a partial order $<$ on $P$ is structured as follows: First we show that the matrix exists if $P$ contains one element. Then we show that the matrix exists if there is a decomposition $\left(I_{1}, I_{2}\right)$ of $P$ such that the matrix exists for the partial ordered sets $I_{1}$ and $I_{2}$ and such that either $I_{1}$ and $I_{2}$ are noncomparable or $I_{2}$ is totally greater than $I_{1}$.

It is not difficult to see that the proof does not imply the existence of the desired matrix for arbitrary partial orders $<$, and thus one is led to the question, for what partial orders does the existence proof work?

This naturally leads to the following recursive definition of those partial orders for which the proof works:

Definition 2.1. A partial order $<$ on $P$ is series parallel if either $P$ contains exactly one element or there is a decomposition $\left(I_{1}, I_{2}\right)$ of $P$ such that the partial orders $<_{I_{1}}$ and $<_{I_{2}}$ are series parallel and such that either $I_{1}$ and $I_{2}$ are noncomparable or $I_{2}$ is totally greater than $I_{1}$.

Obviously a nonrecursive characterization of these partial orders would be preferable. Theorem 2.3, below, states that series-parallel is equivalent to $N$-free, as defined in the following:

Definition 2.2. 1 . An $N$ in $<$ is a four-tuple $(a, b, c, d)$ of elements of $P$ such that the only relations in $<$ between $a, b, c$, and $d$ are: $a<b, c<b$, and $c<d$.

2. A partial order $<$ on $P$ is said to be $N$-free if $P$ contains no $N$ 's.

Thus, in Figure $0,(a, b, c, d)$ is an $N$ in $<$ if the solid-line relations, and only the solid-line relations, hold between $a, b, c$, and $d$. Furthermore, $<$ is $N$-free if whenever each of the solid-line relations holds, then at least one of the dotted-line relations also holds.

It is not difficult to see that stackable (and therefore both trivial and total) orders are $Z$-free.

Theorem 2.3 (see [28]). A partially ordered set is series-parallel if and only if it is $N$-free. 


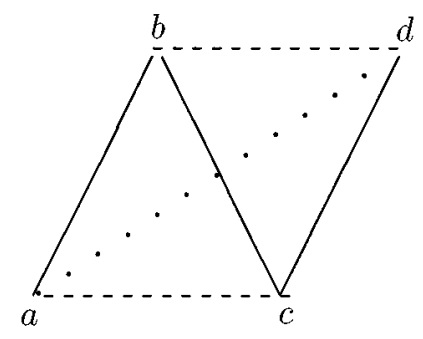

FiguRe 0

Corollary 2.4. If $<$ is $N$-free, then there exists a decomposition $\left(I_{1}, I_{2}\right)$ of $P$ such that $<_{I_{1}}$ and $<_{I_{2}}$ are $N$-free and such that either $I_{1}$ and $I_{2}$ are noncomparable or $I_{2}$ is totally greater than $I_{1}$.

\section{The ALgeBRAIC THEORY}

In this section we present the algebraic theory that we apply to transition systems in the remainder of the paper. The main result of this section, Theorem 3.5, describes conditions under which connection matrices are related by similarity. It is through these similarities that we develop and apply our transition theory in the following sections.

We assume that the reader is familiar with the algebraic connection matrix theory as presented in [6]. Recall,

Definition 3.1. Given $\mathcal{G}$, a graded module braid over $<$, and $C=\{C(p)\}_{p \in P}$, a collection of graded modules, let $\Delta: \bigoplus_{p \in P} C(p) \rightarrow \bigoplus_{p \in P} C(p)$ be a $<$-upper triangular boundary map. Then,

1. if $\mathcal{H} \Delta$, the graded module braid generated by $\Delta$, is isomorphic to $\mathcal{G}$, then $\Delta$ is called a $C$-connection matrix of $\mathcal{G}$;

2. if, furthermore, $C(p)$ is isomorphic to $G(p)$ for each $p \in P$, then $\Delta$ is called a connection matrix of $\mathcal{G}$.

To simplify notation, for $I \in I(<)$ we denote $\bigoplus_{p \in I} C(p)$ by $C(I)$, and the corresponding homology module in $\mathcal{H} \Delta$ by $H(I)$.

Assume $\mathcal{G}, C$, and $\Delta$ are as in Definition 3.1. Let $I$ be an interval in $<$, and set $C_{I}=\{C(p)\}_{p \in I}$. It easily follows that $\Delta(I)$ is a $C_{I}$-connection matrix of $\mathcal{G} \mid<_{I}$. Furthermore, it is not difficult to see that under these circumstances $\mathcal{H}(\Delta(I))$ naturally corresponds to $\mathcal{H} \Delta \mid<_{I}$, and that if $\theta: \mathcal{H} \Delta \rightarrow \mathcal{G}$ is an isomorphism then $\theta\left|<_{I}: \mathcal{H}(\Delta(I)) \rightarrow \mathcal{G}\right|<_{I}$ is too.

In [6] it is shown that if $\mathcal{G}$ is chain complex generated, each $C(p)$ is free, and, for each $p \in P, H(p)$ is isomorphic to $G(p)$ (where $H(p)$ is the homology of the chain complex $C(p)$ with boundary map $\Delta(p)$ ) then the corresponding collection of $C$-connection matrices is not empty.

In this paper we work only with the connection matrices (i.e, not with the more general $C$-connection matrices). We denote the collection of connection matrices of $\mathcal{G}$ by $\mathcal{C M}(\mathcal{G})$.

Connection matrices are not unique. The fact is established via a simple example in [6]; the nonuniqueness can also be seen to be, in part, a consequence of Proposition 3.4, below. The following two propositions, which are easy to verify, are used in 
establishing Proposition 3.4. Recall that if $C=\{C(p)\}_{p \in P}$ and $\Delta: C(P) \rightarrow C(P)$ is <-upper triangular boundary map, then $\mathcal{C} \Delta$ denotes the chain complex braid defined by $\Delta$.

Proposition 3.2. Let $C=\{C(p)\}_{p \in P}$ and $C^{\prime}=\left\{C^{\prime}(p)\right\}_{p \in P}$ be collections of graded modules, and $\Delta: C(P) \rightarrow C(P)$ and $\Delta^{\prime}: C^{\prime}(P) \rightarrow C^{\prime}(P)$ be <-upper triangular boundary maps. If $T: C(P) \rightarrow C^{\prime}(P)$ is <-upper triangular and such that $T \Delta=\Delta^{\prime} T$, then $\mathcal{T}:=\{T(I)\}_{I \in I(<)}$ is a chain map from $\mathcal{C} \Delta$ to $\mathcal{C} \Delta^{\prime}$.

We call $\mathcal{T}$ the chain map induced by $T$, and we call the homology map induced by $\mathcal{T}$, the graded module braid homology map induced by $T$.

The following proposition is a simple matrix-algebra result.

Proposition 3.3. Let $T: C(P) \rightarrow C^{\prime}(P)$ be <-upper triangular, and assume $\left(I_{1}, \ldots, I_{m}\right)$ is a decomposition of $P$. Then

1. $T$ is an isomorphism if and only if $T\left(I_{j}\right)$ is an isomorphism for each $j=$ $1, \ldots, m$ (in particular $T$ is an isomorphism if and only if $T(p)$ is an isomorphism for each $p \in P$ ).

2. If $T$ is an isomorphism with inverse $T^{-1}$, then $T^{-1}$ is $<$-upper triangular and $T^{-1}(I)=T(I)^{-1}$ for each $I \in I(<)$.

The following proposition implies that if $\Delta$ is a connection matrix for a graded module braid $\mathcal{G}$, then any other matrix that is "similar" to $\Delta$ by a $<$-upper triangular isomorphism is also a connection matrix for $\mathcal{G}$. The proof is straightforward and therefore is omitted.

Proposition 3.4. Let $C=\{C(p)\}_{p \in P}$ and $C^{\prime}=\left\{C^{\prime}(p)\right\}_{p \in P}$ be collections of grade modules, and $\Delta: C(P) \rightarrow C(P)$ be a <-upper triangular boundary map. If $T: C(P) \rightarrow C^{\prime}(P)$ is a <-upper triangular isomorphism, then $\Delta^{\prime}:=T \Delta T^{-1}$ is a <-upper triangular boundary map and $\mathcal{T}:=\{T(I)\}_{I \in I(<)}$ is an isomorphism between $\mathcal{C} \Delta$ and $\mathcal{C} \Delta^{\prime}$. In particular, with such $\Delta$ and $T$, if $\Delta \in \mathcal{C M}(\mathcal{G})$.

With $\Delta, \Delta^{\prime}$, and $T$ as in Proposition 3.4, we say that $\Delta$ and $\Delta^{\prime}$ are similar and $T$ is a similarity (isomorphism) between them.

Proposition 3.4 implies that given one connection matrix $\Delta$, others can be computed by using similarity isomorphisms. It is natural to ask if this method is exhaustive, and therefore if all connection matrices can be computed knowing one of them. This question essentially asks if the converse to Proposition 3.4 holds, i.e., if it is true that if $\Delta, \Delta^{\prime} \in \mathcal{C M}(\mathcal{G})$, where $\mathcal{G}$ is a graded module braid over $<$, then there exists a $<$-upper triangular similarity isomorphism between them. In general, the answer is unknown. However, as is shown in Theorem 3.5 below, the answer is affirmative if $<$ is an $N$-free order and $G(p)$ is free and finitely generated for each $p \in P$. In the following sections we show that this partial result is strong enough to establish a broadly applicable transition theory.

Theorem 3.5. Let $\mathcal{G}$ be a graded module braid over an $N$-free order $<$, and assume that $G(p)$ is free and finitely generated for each $p \in P$. If $\Delta, \Delta^{\prime} \in \mathcal{C} \mathcal{M}(\mathcal{G})$, then there exists a <-upper triangular isomorphism $T$ such that $\Delta^{\prime}=T \Delta T^{-1}$.

Theorem 3.5 follows from Theorem 3.8, below; the proof of Theorem 3.5 is presented after that of Theorem 3.8.

Definition 3.6. Let $\Delta$ and $\Delta^{\prime}$ be similar and $T$ be a similarity isomorphism between them. Assume that $\theta: \mathcal{H} \Delta \rightarrow \mathcal{H} \Delta^{\prime}$ is an isomorphism. 
1. If the homology map induced by $T$ equals $\theta(P)$ then we say that $T$ weakly covers $\theta$.

2. If the graded module braid homology map induced by $T$ equals $\theta$ then we say that $T$ covers $\theta$.

The following proposition relates the graded-module-braid boundary map $\partial(J, I)$, in $\mathcal{H} \Delta$, to the map $\Delta(J, I)$, defined by appropriate restriction of $\Delta$.

Proposition 3.7 (see $[6]$ ). If $[\alpha] \in H(J)$, then $\partial(J, I)[\alpha]=[\Delta(J, I) \alpha]$.

Theorem 3.8. Let $C=\{C(p)\}_{p \in P}$ and $C^{\prime}=\left\{C^{\prime}(p)\right\}_{p \in P}$ be collections of free graded modules, $\Delta: C(P) \rightarrow C(P)$ and $\Delta^{\prime}: C^{\prime}(P) \rightarrow C^{\prime}(P)$ be <-upper triangular boundary maps, and $\theta: \mathcal{H} \Delta \rightarrow \mathcal{H} \Delta^{\prime}$ be an isomorphism. Assume that $\left(I_{1}, I_{2}\right)$ is a decomposition of $P$ such that either $I_{1}$ and $I_{2}$ are noncomparable or such that $I_{2}$ is totally greater than $I_{1}$. If for each $i=1,2$ there exists $T_{i}: C\left(I_{i}\right) \rightarrow C^{\prime}\left(I_{i}\right)$, a $<_{I_{i}}$-upper triangular similarity isomorphism between $\Delta\left(I_{i}\right)$ and $\Delta^{\prime}\left(I_{i}\right)$ that weakly covers $\theta \mid<_{i}$, then there exists $T: C(P) \rightarrow C^{\prime}(P), a<$-upper triangular similarity isomorphism between $\Delta$ and $\Delta^{\prime}$ that weakly covers $\theta$ and is such that $T\left(I_{i}\right)=T_{i}$ for each $i=1,2$.

It is unknown if there exists a $T$, as in Theorem 3.8, that covers $\theta$. However, it is only the existence of the map $T$ that is needed in Theorem 3.8 to establish Theorem 3.5 , and therefore to establish the transition theory in the following sections.

Proof of Theorem 3.8. To define $T$, clearly we must set $T\left(I_{i}\right)=T_{i}$, for $i=1,2$, and $T\left(I_{1}, I_{2}\right)=0$. It remains to define $T\left(I_{2}, I_{1}\right)$.

In the case where $I_{1}$ and $I_{2}$ are noncomparable, we must have $T\left(I_{2}, I_{1}\right)=0$. It is not difficult to see that the result holds in this case.

Now consider the case where $I_{2}$ is totally greater than $I_{1}$. We need to define $T\left(I_{2}, I_{1}\right): C\left(I_{2}\right) \rightarrow C\left(I_{1}\right)$. Note that regardless of how $T\left(I_{2}, I_{1}\right)$ is defined, the resulting map $T$ is <-upper triangular, and therefore by Proposition 3.3 is an isomorphism.

Consider the following submodules of $C\left(I_{2}\right)$ :

$$
\begin{aligned}
A & :=\operatorname{Im} \Delta\left(I_{2}\right), \\
A_{1} & :=\left(\Delta\left(I_{2}, I_{1}\right)^{-1}\left(\operatorname{Im} \Delta\left(I_{1}\right)\right)\right) \cap \operatorname{ker} \Delta\left(I_{2}\right), \\
A_{2} & :=\operatorname{ker} \Delta\left(I_{2}\right) .
\end{aligned}
$$

It is not difficult to see that $A \subset A_{1} \subset A_{2}$. Let $B$ be a subspace of $A_{1}$ complementary to $A$, let $C$ be a subspace of $A_{2}$ complementary to $A_{1}$, and let $D$ be a subspace of $C\left(I_{2}\right)$ complementary to $A_{2}$. Note that $D$ is mapped isomorphically to $A$ by $\Delta\left(I_{2}\right)$.

We define $T\left(I_{2}, I_{1}\right)$ on $A, B, C$, and $D$ separately.

For $\delta \in D$ define $T\left(I_{2}, I_{1}\right) \delta=0$.

Now let $\psi$ be an element of a basis for $C$. $\psi \in \operatorname{ker} \Delta\left(I_{2}\right)$, so therefore $T_{2} \psi \in$ $\operatorname{ker} \Delta^{\prime}\left(I_{2}\right)$. We have 


$$
\begin{aligned}
{\left[\Delta^{\prime}\left(I_{2}, I_{1}\right) T_{2} \psi\right] } & =\partial^{\prime}\left(I_{2}, I_{1}\right)\left[T_{2} \psi\right] & & \text { (by Proposition 3.7) } \\
& =\partial^{\prime}\left(I_{2}, I_{1}\right) \theta\left(I_{2}\right)[\psi] & & \left(\text { since } T_{2} \text { induces } \theta\left(I_{2}\right)\right) \\
& =\theta\left(I_{1}\right) \partial\left(I_{2}, I_{1}\right)[\psi] & & (\text { since } \theta \text { is a map between } \\
& =\theta\left(I_{1}\right)\left[\Delta\left(I_{2}, I_{1}\right) \psi\right] & & \text { (by Proposition 3.7) } \\
& =\left[T_{1} \Delta\left(I_{2}, I_{1}\right) \psi\right] & & \left(\text { since } T_{1} \text { induces } \theta\left(I_{1}\right)\right) .
\end{aligned}
$$

Let $\tau \in C^{\prime}\left(I_{1}\right)$ be such that $\Delta^{\prime}\left(I_{1}\right) \tau=T_{1} \Delta\left(I_{2}, I_{1}\right) \psi-\Delta^{\prime}\left(I_{2}, I_{1}\right) T_{2} \psi$. Define $T\left(I_{2}, I_{1}\right) \psi=\tau$.

Let $\beta$ be an element of a basis for $B$. By definition of $B, \Delta\left(I_{2}\right) \beta=0$ and there exists $\gamma \in C\left(I_{1}\right)$ such that $\Delta\left(I_{1}\right) \gamma=\Delta\left(I_{2}, I_{1}\right) \beta$. It is easy to see that $(-\gamma) \oplus \beta \in \operatorname{ker} \Delta$, and therefore $[(-\gamma) \oplus \beta] \in H(P) . \theta(P): H(P) \rightarrow H^{\prime}(P)$; we claim that there exists $\sigma \in C^{\prime}\left(I_{1}\right)$ such that $\left[\sigma \oplus T_{2} \beta\right]=\theta(P)[(-\gamma) \oplus \beta]$. To that end let $\nu \oplus \mu \in C^{\prime}\left(I_{1}\right) \oplus C^{\prime}\left(I_{2}\right)=C^{\prime}(P)$ be such that $[\nu \oplus \mu]=\theta(P)[(-\gamma) \oplus \beta]$. Note that $[\mu]=p^{\prime}\left(P, I_{2}\right)[\nu \oplus \mu]=p^{\prime}\left(P, I_{2}\right) \theta(P)[(-\gamma) \oplus \beta]=\theta\left(I_{2}\right) p\left(P, I_{2}\right)[(-\gamma) \oplus \beta]=$ $\theta\left(I_{2}\right)[\beta]=\left[T_{2} \beta\right]$, and therefore there exists $\rho \in C^{\prime}\left(I_{2}\right)$ such that $\Delta^{\prime}\left(I_{2}\right) \rho=T_{2} \beta-\mu$. Let $\sigma=\nu+\Delta^{\prime}\left(I_{2}, I_{1}\right) \rho$. Note that $\Delta^{\prime}(0 \oplus \rho)=\Delta^{\prime}\left(I_{2}, I_{1}\right) \rho \oplus \Delta^{\prime}\left(I_{2}\right) \rho=(\sigma-\nu) \oplus$ $\left(T_{2} \beta-\mu\right)=\left(\sigma \oplus T_{2} \beta\right)-(\nu \oplus \mu)$. Thus $\left[\sigma \oplus T_{2} \beta\right]=[\nu \oplus \mu]=\theta(P)[(-\gamma) \oplus \beta]$, proving the claim.

With $\beta, \gamma$ and $\sigma$ as above, define $T\left(I_{2}, I_{1}\right) \beta=\sigma+T_{1} \gamma$.

Finally to define $T\left(I_{2}, I_{1}\right)$ on $A$, note that for each $\alpha \in A$, we have $\alpha=\Delta\left(I_{2}\right) \delta$ for a unique $\delta \in D$. For such $\alpha$ define $T\left(I_{2}, I_{1}\right) \alpha=\Delta^{\prime}\left(I_{2}, I_{1}\right) T_{2} \delta-T_{1} \Delta\left(I_{2}, I_{1}\right) \delta$.

Now $T: C(P) \rightarrow C^{\prime}(P)$ is defined. $T$ is <-upper triangular and an isomorphism. We claim that $\Delta^{\prime} T=T \Delta$, and that $T$ induces the homology map $\theta(P): H(P) \rightarrow$ $H^{\prime}(P)$.

It is clear that $\Delta^{\prime} T=T \Delta$ holds on the subspace $C\left(I_{1}\right) \oplus 0 \subset C\left(I_{1}\right) \oplus C\left(I_{2}\right)=$ $C(P)$. To show that $\Delta^{\prime} T=T \Delta$ on $0 \oplus C\left(I_{2}\right)$, we look separately at the subspaces $0 \oplus A, 0 \oplus B, 0 \oplus C$, and $0 \oplus D$ of $0 \oplus C\left(I_{2}\right)$. Thus let $\alpha \in A$ be as above. Then $\alpha=\Delta\left(I_{2}\right) \delta$ for a unique $\delta \in D$.

$$
\begin{aligned}
\Delta^{\prime} T(0 \oplus \alpha) & =\Delta^{\prime}\left(T\left(I_{2}, I_{1}\right) \alpha \oplus T_{2} \alpha\right) \\
& =\Delta^{\prime}\left(\left(\Delta^{\prime}\left(I_{2}, I_{1}\right) T_{2} \delta-T_{1} \Delta\left(I_{2}, I_{1}\right) \delta\right) \oplus T_{2} \alpha\right)
\end{aligned}
$$

(by definition of $T\left(I_{2}, I_{1}\right) \alpha$ )

$$
\begin{gathered}
=\left(\Delta^{\prime}\left(I_{2}, I_{1}\right) T_{2} \Delta\left(I_{2}\right) \delta+\Delta^{\prime}\left(I_{1}\right) \Delta^{\prime}\left(I_{2}, I_{1}\right) T_{2} \delta\right. \\
\left.-\Delta^{\prime}\left(I_{1}\right) T_{1} \Delta\left(I_{2}, I_{1}\right) \delta\right) \oplus \Delta^{\prime}\left(I_{2}\right) T_{2} \alpha \\
=\left(\Delta^{\prime}\left(I_{2}, I_{1}\right) \Delta^{\prime}\left(I_{2}\right) T_{2} \delta+\Delta^{\prime}\left(I_{1}\right) \Delta^{\prime}\left(I_{2}, I_{1}\right) T_{2} \delta\right. \\
\left.-T_{1} \Delta\left(I_{1}\right) \Delta\left(I_{2}, I_{1}\right) \delta\right)
\end{gathered}
$$

(since $T_{1}$ and $T_{2}$ are similarity isomorphisms)

$$
=T_{1} \Delta\left(I_{2}, I_{1}\right) \Delta\left(I_{2}\right) \delta \oplus 0
$$

(since $\Delta^{\prime 2}=0, \Delta^{2}=0$, and $\alpha \in \operatorname{ker} \Delta\left(I_{2}\right)$ )

$$
\begin{aligned}
& =T_{1} \Delta\left(I_{2}, I_{1}\right) \alpha \oplus 0 \\
& =T \Delta(0 \oplus \alpha) .
\end{aligned}
$$


Let $\beta$ be in $B$, and assume that $\gamma$ and $\sigma$ are as above.

$$
\begin{aligned}
\Delta^{\prime} T(0 \oplus \beta) & =\Delta^{\prime}\left(\left(\sigma+T_{1} \gamma\right) \oplus T_{2} \beta\right) & & \text { (by definition of } \left.T\left(I_{2}, I_{1}\right) \beta\right) \\
& =\Delta^{\prime}\left(I_{1}\right) T_{1} \gamma \oplus 0 & & \text { (since } \left.\sigma \oplus T_{2} \beta \in \text { ker } \Delta^{\prime}\right) \\
& =T_{1} \Delta\left(I_{1}\right) \gamma \oplus 0 & & \text { (since } T_{1} \text { is a similarity } \\
& =T_{1} \Delta\left(I_{2}, I_{1}\right) \beta \oplus 0 & & \text { isomorphism) } \\
& =T \Delta(0 \oplus \beta) . & &
\end{aligned}
$$

Now assume $\psi \in C$, and let $\tau$ be as above.

$$
\begin{array}{rlr}
\Delta^{\prime} T(0 \oplus \psi) & \left.=\Delta^{\prime}\left(\tau \oplus T_{2} \psi\right) \quad \text { (by definition of } T\left(I_{2}, I_{1}\right) \psi\right) \\
& =\left(\Delta^{\prime}\left(I_{2}, I_{1}\right) T_{2} \psi+\Delta^{\prime}\left(I_{1}\right) \tau\right) \oplus \Delta^{\prime}\left(I_{2}\right) T_{2} \psi \\
& =T_{1} \Delta\left(I_{2}, I_{1}\right) \psi \oplus T_{2} \Delta\left(I_{2}\right) \psi \quad \text { (by definition of } \tau, \text { and since } \\
& & T_{2} \text { is a similarity isomorphism) } \\
& \left.=T_{1} \Delta\left(I_{2}, I_{1}\right) \psi \oplus 0 \quad \text { (since } \psi \in \operatorname{ker} \Delta\left(I_{2}\right)\right) \\
& =T \Delta(0 \oplus \psi) . &
\end{array}
$$

Finally assume $\delta \in D$, and let $\alpha=\Delta\left(I_{2}\right) \delta$ as above.

$$
\begin{aligned}
\Delta^{\prime} T(0 \oplus \delta)= & \left.\Delta^{\prime}\left(0 \oplus T_{2} \delta\right) \quad \text { (since, by definition, } T\left(I_{2}, I_{1}\right) \delta=0\right) \\
= & \Delta^{\prime}\left(I_{2}, I_{1}\right) T_{2} \delta \oplus \Delta^{\prime}\left(I_{2}\right) T_{2} \delta \\
= & \left(T\left(I_{2}, I_{1}\right) \alpha+T_{1} \Delta\left(I_{2}, I_{1}\right) \delta\right) \oplus T_{2} \Delta\left(I_{2}\right) \delta \\
& \quad \quad \text { by definition of } T\left(I_{2}, I_{1}\right) \alpha, \text { and since } \\
& \left.\quad T_{2} \text { is a similarity isomorphism }\right) \\
= & \left(T\left(I_{2}, I_{1}\right) \Delta\left(I_{2}\right) \delta+T_{1} \Delta\left(I_{2}, I_{1}\right) \delta\right) \oplus T_{2} \Delta\left(I_{2}\right) \delta \\
= & T\left(\Delta\left(I_{2}, I_{1}\right) \delta \oplus \Delta\left(I_{2}\right) \delta\right) \\
= & T \Delta(0 \oplus \delta) .
\end{aligned}
$$

It follows that $\Delta^{\prime} T=T \Delta$.

Finally we show that $T$ induces $\theta(P)$. Let $T_{*}$ be the homology map induced by $T$. We show that $T_{*}=\theta(P)$. Thus let $\omega \in H(P)$. $\omega[\lambda \oplus \kappa]$ for some $\lambda \oplus \kappa \epsilon$ $C\left(I_{1}\right) \oplus C\left(I_{2}\right)=C(P)$. We consider the cases where $\kappa=0$ and $\kappa \neq 0$ separately. Thus assume $\kappa=0$. Then $\lambda \in \operatorname{ker} \Delta\left(I_{1}\right)$, and

$$
\begin{array}{rlr}
T_{*} \omega & =\left[T_{1} \lambda \oplus 0\right] \\
& =i^{\prime}\left(I_{1}, P\right)\left[T_{1} \lambda\right] & \\
& =i^{\prime}\left(I_{1}, P\right) \theta\left(I_{1}\right)[\lambda] & \left(\text { since } T_{1} \text { induces } \theta\left(I_{1}\right)\right) \\
& =\theta(P) i\left(I_{1}, P\right)[\lambda] & \text { (since } \theta \text { is a map between graded } \\
& =\theta(P)[\lambda \oplus 0] & \text { module braids }) \\
& \theta(P) \omega .
\end{array}
$$


Now assume $\kappa \neq 0$. It follows that $\kappa \in A_{1}$ and it is not difficult to see that it suffices to consider $\kappa \in B$ and $\lambda \oplus \kappa=(-\gamma) \oplus \beta$ as above.

$$
\begin{aligned}
T_{*} \omega & =[T((-\gamma) \oplus \beta)] \\
& =\left[\left(T\left(I_{2}, I_{1}\right) \beta+T_{1}(-\gamma)\right) \oplus T_{2} \beta\right] \\
& \left.\left.=\left[\left(\left(\sigma+T_{1} \gamma\right)+T_{1}(-\gamma)\right) \oplus T_{2} \beta\right)\right] \quad \text { (by definition of } T\left(I_{2}, I_{1}\right) \beta\right) \\
& =\left[\sigma \oplus T_{2} \beta\right] \\
& =\theta(P)[(-\gamma) \oplus \beta] \\
& =\theta(P) \omega .
\end{aligned}
$$

It now follows that the homology map induced by $T$ is $\theta(P)$, and the proof of Theorem 3.8 is complete.

Proof of Theorem 3.5. Since $\Delta, \Delta^{\prime} \in \mathcal{C} \mathcal{M}(\mathcal{G})$, it follows that $\mathcal{H} \Delta$ and $\mathcal{H} \Delta^{\prime}$ are isomorphic to $\mathcal{G}$. Thus there exists an isomorphism $\theta: \mathcal{H} \Delta \rightarrow \mathcal{H} \Delta^{\prime} . \Delta: \bigoplus_{p \in P} C(p) \rightarrow$ $\bigoplus_{p \in P} C(p)$ and $\Delta^{\prime}: \bigoplus_{p \in P} C^{\prime}(p) \rightarrow \bigoplus_{p \in P} C^{\prime}(p)$. Each $C(p)$ and $C^{\prime}(p)$ is isomorphic to $G(p)$ and therefore is free. We prove, by induction on the number of elements in $P$, that there exists a <-upper triangular isomorphism $T$ such that $\Delta^{\prime}=T \Delta T^{-1}$ and such that $T$ weakly covers $\theta$.

Consider the case where $P$ has one element. The chain complex $C(P)$ with boundary map $\Delta$ has homology isomorphic to $G(P)$, which is isomorphic to $C(P)$. Since $G(P)$ (and therefore $C(P)$ ) is free and finitely generated, it follows that $\Delta$ must be trivial. Similarly $\Delta^{\prime}$ is trivial. Therefore $H(P)=C(P)$ and $H^{\prime}(P)=$ $C^{\prime}(P)$, and by setting $T=\theta(P)$ it is easy to see that the result holds in this case.

Now assume that the result holds if the number of elements in $P$ is less than $n$, and let $P$ have $n$ elements $(n>1)$. Since $<$ is $Z$-free, Corollary 2.4 implies that there exists a decomposition $\left(I_{1}, I_{2}\right)$ of $P$ such that $<_{I_{1}}$ and $<_{I_{2}}$ are $Z$-free and such that either $I_{1}$ and $I_{2}$ are noncomparable or $I_{2}$ is totally greater than $I_{1}$.

For $i=1,2, \Delta\left(I_{i}\right), \Delta^{\prime}\left(I_{i}\right) \in \mathcal{C} \mathcal{M}\left(\mathcal{G} \mid<_{I_{i}}\right)$. By induction, for $i=1,2$, there exists a $<_{I_{i}}$-upper triangular isomorphism $T_{i}: C\left(I_{i}\right) \rightarrow C^{\prime}\left(I_{i}\right)$ such that $\Delta^{\prime}\left(I_{i}\right)=$ $T_{i} \Delta\left(I_{i}\right) T_{i}^{-1}$ and such that $T_{i}$ weakly covers $\theta \mid<_{I_{i}}$. By Theorem 3.8 there exists a $<$-upper triangular isomorphism $T$ such that $\Delta^{\prime}=T \Delta T^{-1}$ and such that $T$ weakly covers $\theta$.

Thus by induction it now follows that there exists a $<$-upper triangular isomorphism $T$ such that $\Delta^{\prime}=T \Delta T^{-1}$.

\section{Algebraic transition matrices}

Our focus in this section is on the interpretation of Theorem 3.5 in the context of continuation. We begin with the definition of an algebraic transition matrix and then show that these can be used to prove the existence of connecting orbits. This, in turn, can be interpreted as a theorem about global bifurcations.

Let $\mathcal{M}$ be a Morse decomposition. If $<$ is an admissible ordering of $\mathcal{M}$, then the associated homology braid index is denoted by $\mathcal{H}(<)$ and the corresponding collection of connection matrices by $\mathcal{C M}(<)$. Of course for a fixed Morse decomposition, one is interested in the set of connection matrices for the flow defined order. We refer to this as the set of connection matrices for $\mathcal{M}$ and denote it by $\mathcal{C M}(\mathcal{M})$.

The following theorem implies that homology braids and connection matrices are invariant under order preserving continuations. 
Theorem 4.1 (see [7]). Let $\mathcal{M}(S)=\{M(p) \mid p \in \mathcal{P}\}$ be a Morse decomposition for $\Phi_{U}$ with admissible order $<$. Then, given $\lambda_{0}, \lambda_{1} \in U, \mathcal{H}\left(<_{\lambda_{0}}\right)$ and $\mathcal{H}\left(<_{\lambda_{1}}\right)$ are isomorphic and therefore $\mathcal{C M}\left(<_{\lambda_{0}}\right)=\mathcal{C} \mathcal{M}\left(<_{\lambda_{1}}\right)$.

The contrapositive of Theorem 4.1 indicates that if $\mathcal{C M}\left(<_{\lambda_{0}}\right) \neq \mathcal{C M}\left(<_{\lambda_{1}}\right)$ then the admissible orderings $<_{\lambda_{0}}$ and $<_{\lambda_{1}}$ are not related by continuation. Thus if $\mathcal{M}\left(S_{\lambda_{0}}\right)$ and $\mathcal{M}\left(S_{\lambda_{1}}\right)$ are the associated Morse decompositions and $<$ is the partial order on $P$ defining $<_{\lambda_{0}}$ then on any path in $U$ from $\lambda_{0}$ to $\lambda_{1}$ there is a $\gamma$ such that $<$ is not an admissible ordering for the associated Morse decomposition $\mathcal{M}\left(S_{\gamma}\right)$. Therefore there exists a $p<q$ such that the set of connecting orbits, $C\left(M_{\gamma}(p), M_{\gamma}(q)\right)$, associated to the Morse decomposition $\mathcal{M}\left(S_{\lambda_{0}}\right)$, is nonempty. The transition theorem describes conditions under which one can determine algebraically, by examining similarity isomorphisms between connection matrices in $\mathcal{C M}\left(M_{\lambda_{0}}\right)$ and $\mathcal{C M}\left(M_{\lambda_{1}}\right)$, which Morse sets are connected.

Theorem 3.5 indicates that if $\Delta_{\lambda_{0}}, \Delta_{\lambda_{1}} \in \mathcal{C} \mathcal{M}(<)$ then there exists an <-upper triangular isomorphism $T_{\lambda_{1}, \lambda_{0}}$ such that

$$
\Delta_{\lambda_{1}}=T_{\lambda_{1}, \lambda_{0}} \circ \Delta_{\lambda_{0}} \circ T_{\lambda_{1}, \lambda_{0}}^{-1} .
$$

If we wish to express this relation in matrix form then we need to choose bases. Of course, though the spaces $C H_{*}\left(M_{\lambda_{0}}(p)\right)$ and $C H_{*}\left(M_{\lambda_{1}}(p)\right)$ are isomorphic they are different, and hence, there are many potential representations for the identity (7). To eliminate this ambiguity, we shall use the continuation property of the index to uniformly choose bases for all the indices. In particular, if $M_{\lambda_{0}}(p)$ and $M_{\lambda_{1}}(p)$ are related by continuation, then there exists a continuation induced isomorphism (see $[30,13])$

$$
F_{\lambda_{1}, \lambda_{0}}: C H_{*}\left(M_{\lambda_{0}}(p)\right) \rightarrow C H_{*}\left(M_{\lambda_{1}}(p)\right) .
$$

Now assume $M_{\lambda}(p)$ continues over $U \subset \Lambda$ and let $\lambda_{0}, \lambda_{1} \in U$. Let $\mathcal{B}_{\lambda_{0}}$ denote a basis for $C H_{*}\left(M_{\lambda_{0}}(p)\right)$. Then the basis for $C H_{*}\left(M_{\lambda_{1}}(p)\right)$ is defined to be $F_{\lambda_{1}, \lambda_{0}}\left(\mathcal{B}_{\lambda_{0}}\right)$. Observe that under the assumption that $U$ is simply connected, this is a well defined procedure for choosing bases for the Conley indices at different parameter values. The immediate implication of this assumption is that we need only consider <-upper triangular isomorphisms $T_{\lambda_{1}, \lambda_{0}}$ whose diagonal entries are the identity matrix.

Definition 4.2. Let $\mathcal{M}(S)$ be a Morse decomposition which continues with order $<$ over a simply connected set $U \subset \Lambda$. For $\lambda_{0}, \lambda_{1} \in U$ the set of algebraic transition matrices from $\mathcal{C M}\left(\mathcal{M}\left(S_{\lambda_{0}}\right)\right)$ to $\mathcal{C M}\left(\mathcal{M}\left(S_{\lambda_{1}}\right)\right)$ is the collection

$$
\begin{array}{r}
\mathcal{T}_{\lambda_{1}, \lambda_{0}}^{U}:=\left\{T_{\lambda_{1}, \lambda_{0}} \mid T_{\lambda_{1}, \lambda_{0}} \text { is }<\text {-upper triangular, } \Delta_{\lambda_{1}}=T_{\lambda_{1}, \lambda_{0}} \Delta_{\lambda_{0}} T_{\lambda_{1}, \lambda_{0}}^{-1}\right. \\
\text { where } \left.\Delta_{\lambda_{i}} \in \mathcal{C M}\left(\mathcal{M}\left(S_{\lambda_{i}}\right)\right) \text { for } i=0,1\right\}
\end{array}
$$

where the bases for the Conley indices are determined by the continuation isomorphism $F_{\lambda_{1}, \lambda_{0}}$.

Theorem 4.3. Let $<$ be a stackable order associated with the decomposition $\left\{I_{1}, I_{2}, \ldots, I_{n}\right\}$. Let $\mathcal{M}(S)$ be a Morse decomposition which continues with order $<$ over a simply connected set $U \subset \Lambda$. Then, given any $\lambda_{0}, \lambda_{1} \in U$, the set of algebraic transition matrices $\mathcal{T}_{\lambda_{1}, \lambda_{0}}^{U} \neq \varnothing$.

Furthermore, assume that there exists $j$ such that for all $T_{\lambda_{1}, \lambda_{0}} \in \mathcal{T}_{\lambda_{1}, \lambda_{0}}^{U}$, $T_{\lambda_{1}, \lambda_{0}}\left(I_{j-1}, I_{j}\right)$ is nontrivial. Then, there exists a set $V \subset U$ such that $U \backslash V$ consists 
of two components $U_{0}$ and $U_{1}$ such that $\lambda_{0} \in U_{0}, \lambda_{1} \in U_{1}$, and for all $\lambda \in V$,

$$
C\left(M_{\lambda}\left(I_{j}\right), M_{\lambda}\left(I_{j-1}\right)\right) \neq \varnothing .
$$

Proof. We begin by showing that $\mathcal{T}_{\lambda_{1}, \lambda_{0}}^{U} \neq \varnothing$. Note that Theorem 4.1 implies that $\mathcal{C M}\left(<_{\lambda_{0}}\right)=\mathcal{C M}\left(<_{\lambda_{1}}\right)$. Furthermore, $<$ stackable implies that $<$ is $N$-free. Thus, if $\Delta_{\lambda_{i}} \in \mathcal{C M}\left(<_{\lambda_{i}}\right), i=0,1$, then Theorem 3.5 implies there exists $T$ such that $\Delta_{\lambda_{1}}=T \Delta_{\lambda_{0}} T^{-1}$. So $T \in \mathcal{T}_{\lambda_{1}, \lambda_{0}}^{U}$ implying that $\mathcal{T}_{\lambda_{1}, \lambda_{0}}^{U} \neq \varnothing$.

Suppose that $c:[0,1] \rightarrow U$ is a path from $\lambda_{0}$ to $\lambda_{1}$ in $U$ such that for all $s \in[0,1]$, $C\left(M_{c(s)}\left(I_{j}\right), M_{c(s)}\left(I_{j-1}\right)\right)=\varnothing$, containing no Morse decomposition in $\mathcal{C}_{I_{j}, I_{j-1}}$. Let $<^{*}$ be the stackable order having associated decomposition $\left(I_{1}, \ldots, I_{j-2}, I_{j-1} \cup\right.$ $\left.I_{j}, I_{j+1}, \ldots, I_{n}\right)$. It is easy to see that $\mathcal{M}\left(S_{\lambda_{0}}\right)$ and $\mathcal{M}\left(S_{\lambda_{1}}\right)$ are related by a $<*$ order preserving continuation over $c([0,1]) \subset U$. Let $<_{\lambda_{0}}^{*}$ and $<_{\lambda_{1}}^{*}$ be the admissible orderings of $\mathcal{M}\left(S_{\lambda_{0}}\right)$ and $\mathcal{M}\left(S_{\lambda_{1}}\right)$, respectively, corresponding to $<^{*}$. Since $<_{\lambda_{0}}^{*}$ and $<_{\lambda_{1}}^{*}$ are related by continuation;

$$
\mathcal{C M}\left(<_{\lambda_{0}}^{*}\right)=\mathcal{C M}\left(<_{\lambda_{1}}^{*}\right) .
$$

Now $\Delta_{\lambda_{0}} \in \mathcal{C M}\left(\mathcal{M}\left(S_{\lambda_{0}}\right)\right) \subset \mathcal{C M}\left(<_{\lambda_{0}}^{*}\right)$, and $\Delta_{\lambda_{1}} \in \mathcal{C M}\left(\mathcal{M}\left(S_{\lambda_{1}}\right)\right) \subset \mathcal{C M}\left(<_{\lambda_{1}}^{*}\right)$. So $\Delta_{\lambda_{0}}, \Delta_{\lambda_{1}} \in \mathcal{C M}\left(<_{\lambda_{0}}^{*}\right)$. $\mathcal{C M}\left(<_{\lambda_{0}}^{*}\right)$ is the set of connection matrices of the homology index braid $\mathcal{H}\left(<_{\lambda_{0}}^{*}\right)$, a graded module braid over the stackable (and therefore $Z$-free) order $<^{*}$, made up of free and finitely generated homology complexes.

By Theorem 3.5 there exists a $<^{*}$-upper triangular $T$ such that $\Delta_{\lambda_{0}}=T \Delta_{\lambda_{1}} T^{-1}$. Since $<$ is an extension of $<^{*}$ it follows that $T$ is $<$-upper triangular, and therefore $T \in \mathcal{T}$. Since $T$ is $<^{*}$-upper triangular it follows that $T\left(I_{j}, I_{j-1}\right)$ is trivial, contradicting the assumption that $T\left(I_{j}, I_{j-1}\right)$ is nontrivial for every $T \in \mathcal{T}$. Thus if $c$ is any path from $\lambda_{0}$ to $\lambda_{1}$ in $U$, then for at least one parameter value $\lambda \in c((0,1))$

$$
C\left(M_{\lambda}\left(I_{j}\right), M_{\lambda}\left(I_{j-1}\right)\right) \neq \varnothing .
$$

Hence, there exists a set $V$ which separates $\lambda_{0}$ from $\lambda_{1}$.

\section{Applications}

We conclude this paper with some simple applications which are meant to indicate how algebraic transition matrices may be used.

5.1. Bistable attractors. This first example may appear artificial; however, it plays an important step in a description of the dynamics on the global attractors of bistable gradient-like systems. The reader is referred to [18] for a description of the equations. Here we only present the abstract algebraic result.

Let $\varphi_{\lambda}: \mathbf{R} \times X \rightarrow X$ be a continuous family of flows parametrized by $\lambda \in[0,1]$. Let $\Phi: \mathbf{R} \times X \times[0,1] \rightarrow X \times[0,1]$ be the associated parametrized flow. We shall make the following assumptions.

A1 There is a Morse decomposition

$$
\mathcal{M}\left(S_{\lambda}\right)=\left\{M_{\lambda}\left(p^{ \pm}\right) \mid p=0,1, \ldots, P-1\right\} \cup\{M(P)\}
$$

which continues across $[0,1]$.

A2 There is a continuous Lyapunov function

$$
V: S \times[0,1] \rightarrow \mathbf{R}
$$

such that

$$
V\left(M_{\lambda}\left(p^{+}\right), \lambda\right)=V\left(M_{\lambda}\left(p^{-}\right), \lambda\right) .
$$


A3 The Conley indices, using $\mathbf{Z}_{2}$ coefficients, for the Morse sets are given by

$$
C H_{k}\left(M_{\lambda}\left(p^{ \pm}\right)\right) \approx\left\{\begin{array}{ll}
\mathbf{Z}_{2} & \text { if } k=p, \\
0 & \text { otherwise, }
\end{array} \quad p=0, \ldots, P-1,\right.
$$

and

$$
C H_{k}\left(M_{\lambda}(P)\right) \approx \begin{cases}\mathbf{Z}_{2} & \text { if } k=P \\ 0 & \text { otherwise }\end{cases}
$$

A4 Let

$$
\begin{aligned}
\Delta_{0}: \bigoplus_{p=0}^{P-1}\left(C H_{*}\left(M_{0}\left(p^{-}\right)\right)\right. & \left.\oplus C H_{*}\left(M_{0}\left(p^{+}\right)\right)\right) \oplus C H_{*}\left(M_{0}(P)\right) \\
& \rightarrow \bigoplus_{p=0}^{P-1}\left(C H_{*}\left(M_{0}\left(p^{-}\right)\right) \oplus C H_{*}\left(M_{0}\left(p^{+}\right)\right)\right) \oplus C H_{*}\left(M_{0}(P)\right)
\end{aligned}
$$

be a connection matrix for $\mathcal{M}\left(S_{0}\right)$ with $\mathbf{Z}_{2}$ coefficients. Assume that

$$
\Delta_{0}=\left[\begin{array}{cccccccc}
0 & 0 & 1 & 1 & 0 & 0 & \ldots & 0 \\
0 & 0 & 1 & 1 & 0 & 0 & \ldots & 0 \\
0 & 0 & 0 & 0 & 1 & 1 & \ldots & 0 \\
0 & 0 & 0 & 0 & 1 & 1 & \ldots & 0 \\
& & & & & & \ddots & \vdots \\
& \vdots & & & & & & 1 \\
& & & & & & & 1 \\
0 & 0 & & \ldots & & & & 0
\end{array}\right] .
$$

Theorem 5.1. Given $\mathrm{A} 1-\mathrm{A} 4$, if $\Delta_{\lambda}$ denotes a connection matrix for $\mathcal{M}\left(S_{\lambda}\right)$, then for all $\lambda \in[0,1]$,

$$
\Delta_{\lambda}=\Delta_{0} .
$$

Proof. Let $<_{\lambda}$ denote the flow defined order for $\mathcal{M}\left(S_{\lambda}\right)$. A2 implies that $p^{+}$and $p^{-}$are not related by $<_{\lambda}$ for all $\lambda \in[0,1]$. A4 implies that

$$
0^{ \pm}<_{0} 1^{ \pm}<_{0} \cdots<_{0} P-1^{ \pm}<_{0} P \text {. }
$$

Let $\mathcal{T}_{0,0}$ denote the set of algebraic transition matrices at $\lambda=0$. Then it is easy to check that the identity matrix is the only $<_{0}$-upper triangular matrix, and hence, the only element of $\mathcal{T}_{0,0}$. This implies that $\Delta_{0}$ is the unique connection matrix at $\lambda=0$.

Observe that if we knew that $\mathcal{M}(S)$ continued over [0,1] while preserving an order, then by the previous argument we would be done. We shall now prove this using an induction argument.

By Theorem 1.1 there exist intervals

$$
\left\{\left(\alpha_{k}, \beta_{k}\right) \mid \alpha_{k}<\beta_{k-1}<\alpha_{k+1}<\beta_{k}, k=1, \ldots, K\right\}
$$

which cover $[0,1]$ such that $\mathcal{M}(S)$ continues with order $<^{k}$ over $\left(\alpha_{k}, \beta_{k}\right)$.

Observe that on $\left[0, \beta_{1}\right) \subset\left(\alpha_{1}, \beta_{1}\right),<_{0}$ is an extension of any order which satisfies A2. Thus, for every $\lambda \in\left[0, \beta_{1}\right), \mathcal{T}_{\lambda, 0}=\{i d\}$. This, in turn, implies that $\Delta_{\lambda}=\Delta_{0}$ and $<^{1}=<_{0}$. 
Now for some $k \geq 1$, assume that $<^{k}=<_{0}$ and that for $\lambda \in\left(\alpha_{k}, \beta_{k}\right), \Delta_{\lambda}=\Delta_{0}$. We need to show that $<^{k+1}=<_{0}$. Observe that on $\left(\alpha_{k+1}, \beta_{k}\right),<^{k}$ is an extension of any order which satisfies A2. In particular, $<^{k}$ is an extension of $<^{k+1}$ which is admissible over all of $\left(\alpha_{k+1}, \beta_{k+1}\right)$. Thus, given $\lambda \in\left(\alpha_{k}, \beta_{k}\right)$ and for all $\mu \in$ $\left(\alpha_{k+1}, \beta_{k+1}\right), \mathcal{T}_{\mu, \lambda}=\{i d\}$. Therefore, for all $\mu \in\left(\alpha_{k+1}, \beta_{k+1}\right), \Delta_{\mu}=\Delta_{\lambda}=\Delta_{0}$ and hence, $<^{k+1}=<^{k}=<_{0}$.

5.2. Travelling waves for gradient systems. Consider a system of reaction diffusion equations of the form

$$
u_{t}=D u_{x x}+\nabla F(u)
$$

where $x, t \in \mathbf{R}, u \in \mathbf{R}^{n}, D$ is a diagonal matrix with positive diagonal entries $\left\{d_{1}, d_{2}, \ldots, d_{n}\right\}$, and $F: \mathbf{R}^{n} \rightarrow \mathbf{R}$ is sufficiently smooth. A travelling wave solution of (8) has the form $u(x, t)=u(x-\theta t)=u(\tau)$. It is easy to check that such a solution must satisfy the following system of ordinary differential equations:

$$
\dot{u}=v, \quad D \dot{v}=-\theta v-\nabla F(u) .
$$

We shall restrict our attention to positive wave speeds, that is $\theta>0$. It is easy to check that the function

$$
H(u, v):=\frac{1}{2}\langle D v, v\rangle+F(u)
$$

is a Lyapunov function for (9) in the sense that along solutions

$$
\frac{d H}{d \tau}=-\theta\|v\|^{2} .
$$

Thus, bounded trajectories (which are the ones of interest) of (9) consist of equilibria and connecting orbits between equilibria. To make our analysis of the orbits manageable we use the following assumptions.

B1 $F$ has a finite number of nondegenerate critical points, $\left\{M_{p} \mid p=1, \ldots, P\right\}$ where $F\left(M_{p}\right)>F\left(M_{p-1}\right)$.

B2 There exists $Q_{0}$ such that if $Q<Q_{0}$, then the level surfaces $\left\{u \in \mathbf{R}^{n} \mid F(u)=\right.$ $Q\}$ are convex and $\lim _{\|u\| \rightarrow \infty} F(u)=-\infty$.

Let $S_{\theta}$ denote the set of bounded solutions to (9) at wave speed $\theta$. In [1] it is shown that under assumption $\mathrm{B} 2, S_{\theta}$ is a compact set. Also, observe that given a critical point $M_{p}$ of $F,\left(M_{p}, 0\right)$ is an equilibrium for (9). Combining this with the existence of a Lyapunov function gives the following result.

Lemma 5.2.

$$
\mathcal{M}(S):=\left\{M(p)=\left(M_{p}, 0\right) \mid p=1, \ldots, P\right\}
$$

is a Morse decomposition which continues with order

$$
1<2<\cdots<P-1<P
$$

over $\theta \in(0, \infty)$.

It is reasonably straightforward to compute the Conley indices of these objects which gives the following proposition.

Proposition 5.3. Using $\mathbf{Z}_{2}$ coefficients, the Conley indices of $S_{\theta}$ and the Morse sets are as follows. 
1.

$$
C H_{k}\left(S_{\theta} ; \mathbf{Z}_{2}\right) \approx \begin{cases}\mathbf{Z}_{2} & \text { if } k=n, \\ 0 & \text { otherwise. }\end{cases}
$$

2. Let $\eta$ denote the number of negative eigenvalues of $D^{2} F\left(M_{p}\right)$ where $M_{p}$ is a critical point of $F$. Then

$$
C H_{k}\left(M(p) ; \mathbf{Z}_{2}\right) \approx \begin{cases}\mathbf{Z}_{2} & \text { if } k=\eta, \\ 0 & \text { otherwise. }\end{cases}
$$

For the purpose of describing the connection matrices for these Morse decompositions it is useful to partition the Morse sets according to their index. Let

$$
J_{k}:=\left\{M(p) \mid C H_{k}(M(p)) \not \nsim 0\right\} .
$$

Let $\Delta_{\theta}$ denote a connection matrix for $\mathcal{M}\left(S_{\theta}\right)$ and let

$$
\Delta_{\theta}\left(J_{m}, J_{k}\right): \bigoplus_{p \in J_{k}} C H_{*}(M(p)) \rightarrow \bigoplus_{p \in J_{m}} C H_{*}(M(p))
$$

denote the corresponding submatrix. Then, it follows directly from the definition of a connection matrix that the only nonzero elements of $\Delta_{\theta}$ lie in $\Delta_{\theta}\left(J_{k-1}, J_{k}\right)$.

The final general remark which we wish to make concerns the computation of the connection matrix for large values of $|\theta|$. We begin with the observation that B2 implies that the set of bounded solutions, $K$, to the gradient system

$$
\dot{u}=-\nabla F(u)
$$

is compact and that

$$
\mathcal{M}(K):=\left\{M_{p} \mid p=1, \ldots, P\right\}
$$

is a Morse decomposition with an admissible order $1<2<\cdots<P$. Furthermore,

$$
C H_{*}(M(p)) \approx C H_{*}\left(M_{p}\right) .
$$

Now assume that the system (10) is Morse-Smale. Then, it follows from [2] (see also $[19,25])$ that for $|\theta|$ sufficiently large $\Delta_{\theta}$ is isomorphic to the connection matrix associated with

$$
\mathcal{M}(K):=\left\{M_{p} \mid p=1, \ldots, P\right\} .
$$

To be more precise, given a gradient Morse-Smale system, Reineck [27] showed that connection matrices are unique. Let $\Delta_{\text {grad }}$ denote the connection matrix for the Morse decomposition (11). Then, there exists a conjugation between $\Delta_{\text {grad }}$ and $\Delta_{\theta}$ for $|\theta|$ sufficiently large. In an abuse of notation we shall write this as

$$
\Delta_{\text {grad }}=\Delta_{\theta}
$$

We could continue in this general vein for some time, but for the sake of clarity we now restrict our attention to the case where $n=2$ and $P=5$.

The first question to address is what are the possible sets $J_{k}$ ? Since $n=2$, $k \leq 2$. Furthermore, by Proposition 5.3 the kernel of $\Delta_{\theta}$ modulo the image of $\Delta_{\theta}$ must be isomorphic $\mathbf{Z}_{2}$. We leave it to the reader to check that this implies that the only possible partitions are as follows:

(1) $J_{1}=\{1,2\}$ and $J_{2}=\{3,4,5\}$,

(2) $J_{1}=\{1,3\}$ and $J_{2}=\{2,4,5\}$,

(3) $J_{0}=\{1\}, J_{1}=\{2,3\}$ and $J_{2}=\{4,5\}$, 
(4) $J_{0}=\{1,2\}, J_{1}=\{3,4\}$ and $J_{2}=\{5\}$,

(5) $J_{0}=\{1,3\}, J_{1}=\{2,4\}$ and $J_{2}=\{5\}$.

Because of the similarities between (1) and (2) and between (4) and (5), we shall only consider the cases (1), (3), and (4).

Before beginning the computations, it is worth reflecting on what is being computed. First, observe that since the connection matrix is a degree -1 operator, it detects connecting orbits between critical points whose unstable manifolds differ in dimension by one. Thus, one expects that these connections occur, at least generically, via transverse intersections of the appropriate stable and unstable manifolds. This, in turn, implies that the connections persist under perturbations. With this in mind, we adopt the point of view that connection matrices are directly computable. M. Eidenschink and Mischaikow [3] are currently developing numerical algorithms for computing connection matrices for systems of ordinary differential equations. This, combined with the work of M. Mrozek and Mischaikow [22, 23], provides a technique for rigorously determining the connection matrices for fixed choices of $\theta$. In the language of travelling waves, the connections found via the connection matrix correspond most closely to Fischer waves.

The algebraic transition matrices are degree 0 operators, and hence, detect connecting orbits between fixed points with unstable manifolds of the same dimension. Again, generically one expects that these connections occur for only a discrete set of parameter values. Therefore, we adopt the point of view that these connections cannot be computed directly since the wave speed for which they occur is not known. These type of connections correspond to bistable waves.

With this in mind our strategy is as follows. Having fixed $n, P$, and the sets $J_{p}$, we compute all connection matrices for the order $<$ determined by the Lyapunov function $H$. By Theorem 3.5, this can be done by finding one connection matrix (we do this by studying the gradient system (10)) and then conjugating by all algebraic transition matrices.

However, we wish to do this last step in a more refined manner and so introduce the following notion. An algebraic transition matrix $T$ is elementary if it has exactly one off diagonal entry which is nonzero. It is easy to check that all algebraic transition matrices can be obtained as products of elementary transition matrices. Thus beginning with our one connection matrix we generate all other connection matrices by conjugation by products of elementary transition matrices. We display this construction in the form of a graph, called an elementary transition graph, in which the vertices are connection matrices and the edges are elementary transition matrices.

5.2.1. $J_{1}=\{1,2\}$ and $J_{2}=\{3,4,5\}$. As was mentioned above we begin our analysis by considering the connection matrix $\Delta_{\text {grad }}$ associated with the Morse decomposition (11) for the gradient system (10). It is easy to check that the only nonzero block in $\Delta_{\text {grad }}$ is

$$
\Delta_{\text {grad }}\left(J_{1}, J_{2}\right):=\left[\begin{array}{lll}
\Delta_{3,1} & \Delta_{4,1} & \Delta_{5,1} \\
\Delta_{3,2} & \Delta_{4,2} & \Delta_{5,2}
\end{array}\right]
$$

where

$$
\Delta_{p, q}: C H_{2}(M(q)) \rightarrow C H_{1}(M(p)) .
$$

Observe that the fixed points $M_{p}, p=1,2$, are saddles and hence have onedimensional stable manifolds. Furthermore, by the Morse-Smale assumption and 
B2, these stable manifolds are heteroclinic orbits from the repelling fixed points $M_{p}, p=3,4,5$. By [12] we can conclude that

$$
\Delta_{p, q}=\text { number of connecting orbits from } M_{q} \text { to } M_{p} \bmod 2 .
$$

Since each $M_{p}, p=1,2,3$, must have exactly two heteroclinic orbits associated with it the sum of the row entries of $\Delta_{\text {grad }}\left(J_{1}, J_{2}\right)$ equals $0 \bmod 2$. Since the rank of $\Delta_{\text {grad }}$ is two the rank of $\Delta_{\text {grad }}\left(J_{1}, J_{2}\right)$ is two, and hence each row contains two nonzero entries. This analysis provides necessary conditions for the connection matrices for the gradient system. To see that all such matrices can occur as connection matrices consider a nonlinearity $F$ of the form

$$
F\left(u_{1}, u_{2}\right)=f\left(u_{1}\right)-u_{2}^{2} .
$$

Clearly, all the critical points of $F$ lie on the $u_{1}$ axis. It is easy to determine the connection matrices for systems of this form and by appropriate arrangements of the critical points all connection matrices can be realized.

Having determined the connection matrices $\Delta_{\text {grad }}$ we now use the relation (12) to determine the connection matrices for large $\theta$.

We now need to determine all the possible elementary transition matrices. However, if $T$ is an algebraic transition matrix then it must be of the form

$$
T=\left[\begin{array}{ccccc}
1 & T_{1,2} & 0 & 0 & 0 \\
0 & 1 & 0 & 0 & 0 \\
0 & 0 & 1 & T_{3,4} & T_{3,5} \\
0 & 0 & 0 & 1 & T_{4,5} \\
0 & 0 & 0 & 0 & 1
\end{array}\right] .
$$

So let $E_{p, q}$ denote the elementary transition matrix where the only nonzero off diagonal entry is $T_{p, q}=1$. The resulting elementary transition graph is shown in Figure 1.

It is reasonable to ask what is gained by these computations. Recall that we argued that connection matrices are computable objects. Thus, for a given nonlinearity we foresee choosing two wave speeds and computing the connection matrices. For these two wave speeds the $\Delta\left(J_{1}, J_{2}\right)$ entries will be given by one of the "vertices" of the elementary transition graph. If these submatrices are different, then they can be connected by a series of edges on the graph. For example, if one matrix lies in the lower half of the graph and the other lies in the upper half, then any path which connects these two matrices contains an edge labelled $E_{1,2}$. Hence, in this case, one could conclude that for an intermediate wave speed there exists a travelling wave from $\left(M_{2}, 0\right)$ to $\left(M_{1}, 0\right)$.

5.2.2. $J_{0}=\{1,2\}, J_{1}=\{3,4\}$, and $J_{2}=\{5\}$. Again, we begin the analysis by considering the gradient system (10).

Proposition 5.4. The connection matrix

$$
\Delta_{\text {grad }}: \bigoplus_{p=0}^{5} C H_{*}\left(M_{p}\right) \rightarrow \bigoplus_{p=0}^{5} C H_{*}\left(M_{p}\right)
$$




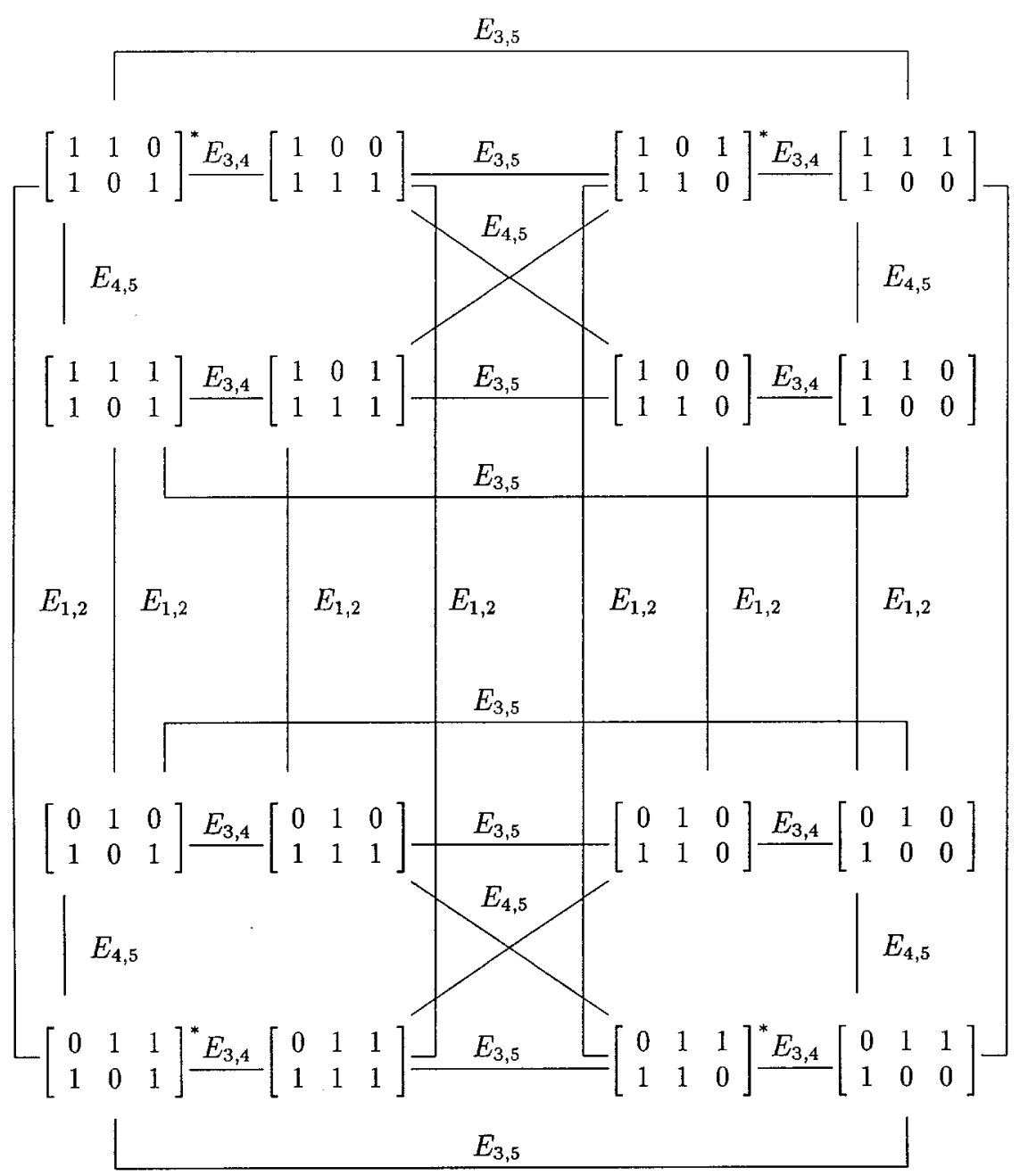

Figure 1. The elementary transition graph for $J_{1}=\{1,2\}$ and $J_{2}=\{3,4,5\}$. Only the entries in $\Delta\left(J_{1}, J_{2}\right)$ are indicated, since the other entries in $\Delta$ are zero. The $*$ indicate connection matrices which can occur for $\theta$ very large.

is of the form

$$
\Delta_{\text {grad }}=\left[\begin{array}{ccccc}
0 & 0 & \alpha & \beta & 0 \\
0 & 0 & \delta & \gamma & 0 \\
0 & 0 & 0 & 0 & 0 \\
0 & 0 & 0 & 0 & 0 \\
0 & 0 & 0 & 0 & 0
\end{array}\right]
$$

where $\alpha \delta+\beta \gamma=1$. 


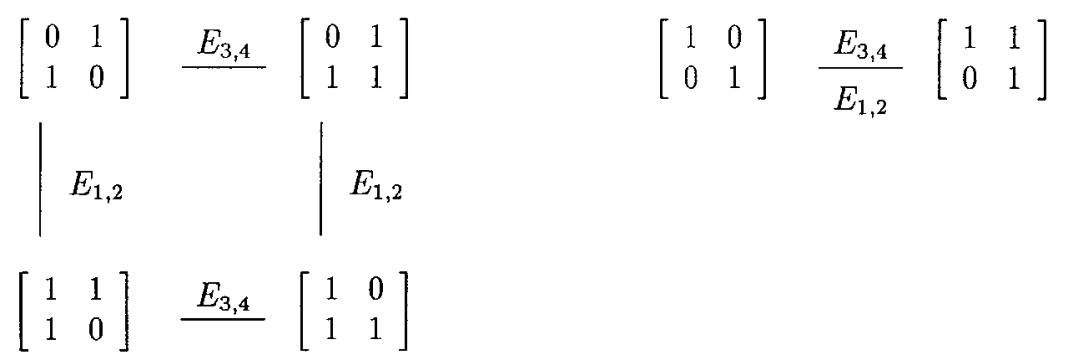

FiguRE 2. The elementary transition graphs for $J_{0}=\{1,2\}, J_{1}=$ $\{3,4\}$, and $J_{2}=\{5\}$. Only the entries in $\Delta\left(J_{0}, J_{1}\right)$ are indicated, since the other entries in $\Delta$ are zero.

Proof. Since the connection matrix is a degree -1 operator, $\Delta_{\text {grad }}$ must be of the form

$$
\left[\begin{array}{lllll}
0 & 0 & \alpha & \beta & 0 \\
0 & 0 & \delta & \gamma & 0 \\
0 & 0 & 0 & 0 & a \\
0 & 0 & 0 & 0 & b \\
0 & 0 & 0 & 0 & 0
\end{array}\right]
$$

As was indicated in the previous case, $M_{3}$ and $M_{4}$ are saddle points and each branch of the stable manifold represents a heteroclinic orbit from $M_{5}$. Therefore, $a=b=0$. But the rank of $\Delta_{\text {grad }}$ equals 2, and hence, $\alpha \delta+\beta \gamma=1$.

We now use the relation (12) to conclude that we have calculated the potential connection matrices for the travelling wave problem for large wave speed. Observe that the only elementary transition matrices are $E_{1,2}$ and $E_{3,4}$. The corresponding elementary transition graphs are given in Figure 2. We leave it to the reader to check that each elementary transition graph can be realized by an appropriate connection matrix of a gradient flow in the plane.

5.2.3. $J_{0}=\{1\}, J_{1}=\{2,3\}, J_{2}=\{4,5\}$. Using similar arguments as before, we can use the gradient system to determine that for large wave speed the connection matrix

$$
\Delta: \bigoplus_{p=0}^{5} C H_{*}(M(p)) \rightarrow \bigoplus_{p=0}^{5} C H_{*}(M(p))
$$

must be of the following type

$$
\left[\begin{array}{lllll}
0 & 1 & 1 & 0 & 0 \\
0 & 0 & 0 & 1 & 1 \\
0 & 0 & 0 & 1 & 1 \\
0 & 0 & 0 & 0 & 0 \\
0 & 0 & 0 & 0 & 0
\end{array}\right],\left[\begin{array}{lllll}
0 & 1 & 0 & 0 & 0 \\
0 & 0 & 0 & 0 & 0 \\
0 & 0 & 0 & 1 & 1 \\
0 & 0 & 0 & 0 & 0 \\
0 & 0 & 0 & 0 & 0
\end{array}\right],\left[\begin{array}{lllll}
0 & 0 & 1 & 0 & 0 \\
0 & 0 & 0 & 1 & 1 \\
0 & 0 & 0 & 0 & 0 \\
0 & 0 & 0 & 0 & 0 \\
0 & 0 & 0 & 0 & 0
\end{array}\right] .
$$

Since the only possible elementary transition matrices are $E_{2,3}$ and $E_{4,5}$ it is easy to check that the elementary transition graphs are as indicated in Figure 3. 


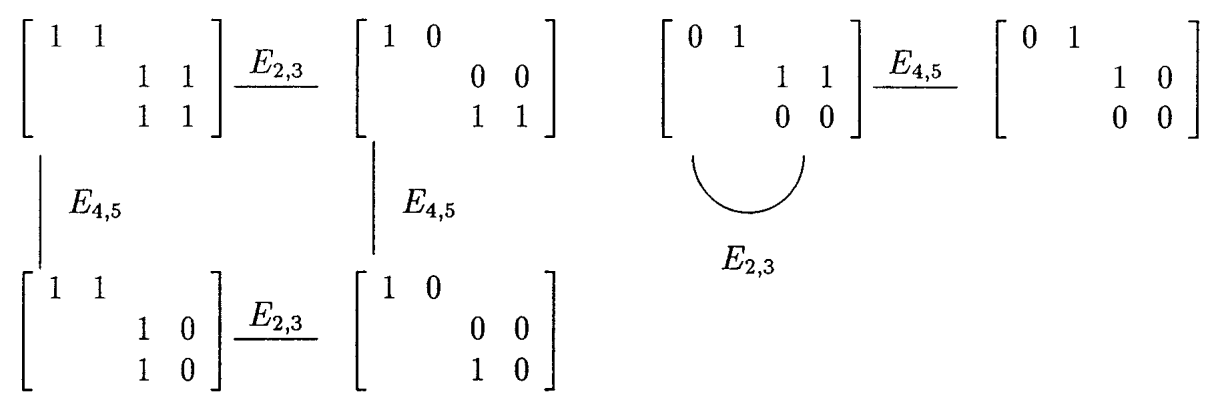

Figure 3. The elementary transition graphs for $J_{0}=\{1\}, J_{1}=$ $\{2,3\}$, and $J_{2}=\{4,5\}$. Only the entries in $\Delta\left(J_{0}, J_{1}\right)$ and $\Delta\left(J_{1}, J_{2}\right)$ are indicated, since the other entries in $\Delta$ are zero.

\section{REFERENCES}

[1] C. Conley, Isolated Invariant Sets and the Morse Index, CBMS Lecture Note 38 A.M.S. Providence, RI, 1978. MR 80f:58023

[2] C. Conley and P. Fife, Critical manifolds, travelling waves, and an example from population genetics, J. Math. Bio. 14 (1982), 159-176. MR 83m:92044

[3] M. Eidenschink and K. Mischaikow, A numerical algorithm for finding isolating neighborhoods, in progress.

[4] B. Fiedler and K. Mischaikow, Dynamics of bifurcations for variational problems with $O(3)$ equivariance: a Conley index approach, Arch. Rational Mech. Anal. 119 (1992), 145-196. MR 93h: 58110

[5] R. Franzosa, Index filtrations and the homology index braid for partially ordered Morse decompositions, Trans. Amer. Math. Soc. 298 (1986), 193-213. MR 88a:58121

[6] - The continuation theory for Morse decompositions and connection matrices, Trans. Amer. Math. Soc. 310 (1988), 781-803. MR 90g:58111

[7] _ The connection matrix theory for Morse decompositions, Trans. Amer. Math. Soc. 311 (1989), 561-592. MR 90a:58149

[8] R. Franzosa and K. Mischaikow, The connection matrix theory for semiflows on (not necessarily locally compact) metric spaces, J.D.E., 71 (1988), 270-287. MR 89c:54078

[9] T. Gedeon and K. Mischaikow, Structure of the global attractor of cyclic feedback systems, Dyn. Diff. Eqs. (to appear).

[10] H. Kokubu, K. Mischaikow and H. Oka, Existence of infinitely many connecting orbits in a singularly perturbed ordinary differential equation, Nonlinearity 9 (1996), 1263-1280. CMP 97:03

[11] J. Mallet-Paret, Morse decompositions for delay-differential equations, JDE 72 (1988), 270315. MR 89m:58182

[12] C. McCord, The connection map for attractor-repeller pairs, Trans. Amer. Math. Soc. 307 (1988), 195-203. MR 89f:58086

[13] C. McCord and K. Mischaikow, Connected simple systems, transition matrices and heteroclinic bifurcations, Trans. Amer. Math. Soc. 333 (1992), 397-422. MR 92k:58193

[14] - On the global dynamics of attractors for scalar delay equations, J. Amer. Math. Soc. 9 (1996), 1095-1133. MR 96m:34139

[15] Singular and Topological Transition Matrices in the Conley Index Theory, preprint.

[16] K. Mischaikow, Existence of generalized homoclinic orbits for one parameter families of flows, Proc. AMS 103 (1988), 59-68. MR 89k:58147

[17] — Transition Systems, Proc. Roy. Soc. Edin. 112A (1989), 155-175. MR 91b:58215

[18] _ Global asymptotic dynamics of gradient-like bistable equations, SIAM J. Math. Anal., to appear.

[19] K. Mischaikow and V. Hutson, Travelling waves for mutualist species, SIAM J. Math. Anal. 24 (1993), 987-1008. MR 94m:92014 
[20] K. Mischaikow and Y. Morita, Dynamics on the Global Attractor of a Gradient Flow Arising from the Ginzburg-Landau Equation, JJIAM 11 (1994), 185-202. MR 95h:58082

[21] R. Moeckel, Morse decompositions and connection matrices, Erg. Th. \& Dyn. Sys. 8 (1988), 227-250. MR 89k:58249

[22] K. Mischaikow and M. Mrozek, Chaos in the Lorenz Equations: a Computer-Assisted Proof, Bull. A.M.S. (N.S.) 32 (1995), 66-72. MR 95e:58121

[23] _ Chaos in the Lorenz Equations: the details, in preparation.

[24] _ Singular Index Pairs, preprint.

[25] K. Mischaikow and J. Reineck, Travelling waves in predator-prey systems, SIAM J. Math. Anal. 24 (1993), 1179-1214. MR 94m:92014

[26] J. Reineck, Connecting orbits in one-parameter families of flows, Erg. Th. \& Dyn. Sys. 8 (1988), 359-374. MR 89i:58128

[27] _ The connection matrix in Morse-Smale flows, Trans. AMS 322 (1990), 523-544. MR 91c:58066

[28] I. Rival, Stories about order and the letter $N$ (en), Contemporary Math., 57, A.M.S., Providence, RI, 1986. MR 87k:06006

[29] K. P. Rybakowski, The Homotopy Index and Partial Differential Equations, Universitext, Springer-Verlag, 1987. MR 89d:58025

[30] D. Salamon, Connected simple systems and the Conley index of isolated invariant sets, Trans. Amer. Math. Soc. 291 (1985), 1-41. MR 87e:58182

[31] J. Smoller, Shock Waves and Reaction-Diffusion Equations, Springer-Verlag, New York, 1983. MR 84d:35002

Department of Mathematics, University of Maine, Orono, Maine 04469

E-mail address: franzosa@gauss.umemat.maine.edu

Center for Dynamical Systems and Nonlinear Studies, School of Mathematics, Georgia Institute of Technology, Atlanta, Georgia 30332

E-mail address: mischail@math.gatech.edu 